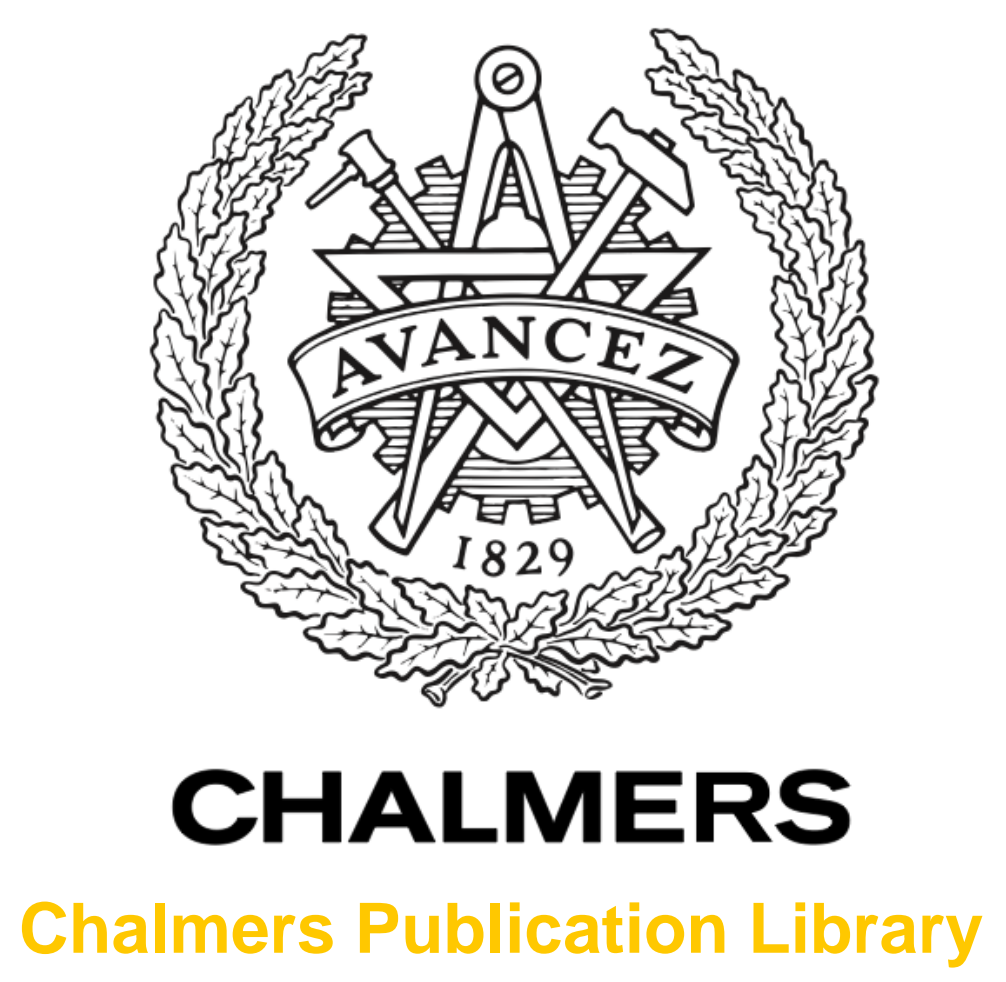

\title{
Resonance Micro-Raman Investigations of the Rat Medial Preoptic Nucleus: Effects of a Low-Iron Diet on the Neuroglobin Content
}

This document has been downloaded from Chalmers Publication Library (CPL). It is the author's version of a work that was accepted for publication in:

Applied Spectroscopy (ISSN: 0003-7028)

Citation for the published paper:

Ramser, K. ; Malinina, E. ; Candefjord, S. (2012) "Resonance Micro-Raman Investigations of the Rat Medial Preoptic Nucleus: Effects of a Low-Iron Diet on the Neuroglobin Content". Applied Spectroscopy, vol. 66(12), pp. 1454-1460.

http://dx.doi.org/10.1366/12-06670

Downloaded from: http://publications.lib.chalmers.se/publication/170757

Notice: Changes introduced as a result of publishing processes such as copy-editing and formatting may not be reflected in this document. For a definitive version of this work, please refer to the published source. Please note that access to the published version might require a subscription. 


\title{
Resonance microRaman investigations of the rat medial preoptic nucleus: effects of a low iron diet on the neuroglobin content
}

Kerstin Ramser ${ }^{1,2}$, Evgenya Malinina ${ }^{1,5}$ and Stefan Candefjord ${ }^{1,2,3,4}$

${ }^{1}$ Department of Computer Science, Electrical and Space Engineering, Luleå University of Technology, SE-971 87 Luleå, Sweden

${ }^{2}$ Centre for Biomedical Engineering and Physics, Luleå University of Technology and Umeå University, Luleå and Umeå, Sweden

${ }^{3}$ Signals and Systems, Chalmers University of Technology, 412 96, Gothenburg, Sweden

${ }^{4}$ MedTech West, Sahlgrenska University Hospital, Blå Stråket 7, 413 45, Gothenburg, Sweden

${ }^{5}$ Department of Integrative Medical Biology, Umeå University, 90187 Umeå, Sweden

kerstin.ramser@ltu.se

Keywords: resonance Raman spectroscopy, fresh brain tissue, medial preoptic nucleus, neuroglobin, hemoglobin

\begin{abstract}
The aim of the study was to investigate the medial preoptic nucleus (MPN) of the anterior hypothalamus by resonance Raman spectroscopy $(514.5 \mathrm{~nm})$ to see if it is possible to enhance the Raman scattering of hemoproteins in fresh brain tissue slices. The resonance effect was compared to near infrared (NIR) Raman spectra. Two groups of male Sprague Dawley rats were studied: one control group under normal diet and one group under a low-iron diet to evoke iron deficiency. Each group consisted of four rats, 38 to 41 days old. The diet lasted for 11, 12 and 15 days. The MPN regions of brain tissue slices were analyzed by monitoring raw and preprocessed mean data, by cluster analysis, and by deriving difference spectra from preprocessed mean spectra. Cluster analysis of the resonance Raman spectra could identify different hemoprotein groups, namely hemoglobin $(\mathrm{Hb})$ and neuroglobin $(\mathrm{Ngb})$. Spectra from randomly distributed spots revealed high $\mathrm{Hb}$ content, whereas $\mathrm{Ngb}$ was evenly distributed in the MPN. The difference spectra showed a decrease of the Ngb and lipid content for the animals under low iron diet. The Ngb decrease was approximately $20 \%$. The data shows that resonance Raman spectroscopy is well suited to study hemoproteins in fresh brain tissue.
\end{abstract}




\section{Introduction}

Raman spectroscopy gives detailed information on biochemical content and structure and has found many biomedical applications. The great benefit of the technique is that no staining is necessary. Due to that, it is possible to combine Raman spectroscopy with other methods, like electrophysiological recordings, where it is not feasible to work with stained samples. Especially near infrared (NIR) Raman spectroscopy has shown to be valuable since excitation in this wavelength region can prevent or at least diminish photo-induced effects on fragile biological samples. For instance, NIR Raman spectroscopy is useful for detecting various cancer types. ${ }^{1-9}$ NIR Raman spectroscopy has been successfully applied for the classification of porcine brain tissue by a single Raman fiber optical probe, ${ }^{10}$ for the detection of acute brain injury in mouse brain blood serum, ${ }^{11}$ and for brain tumor classification in vitro and in vivo. ${ }^{12-15}$

Generally, Raman scattering is a weak process, only one photon in a million is Raman scattered. It is possible to enhance the signal from certain compounds by resonance Raman spectroscopy. If a wavelength close to an electronic transition of a specific compound is chosen the Raman effect can be enhanced dramatically. Resonance Raman spectroscopy has been used to identify carotenoids in human skin in vivo, ${ }^{16}$ to distinguish hemoproteins in cardiomyocytes and fibrotic tissue with principal component analysis, ${ }^{17}$ and to analyze the oxygen saturation in microvessels of anesthetized rats. ${ }^{18}$ In fact, resonance Raman spectroscopy has been used for four decades to study hemoproteins. ${ }^{19}$ The resonance effect has shown to be strong enough that the hemoprotein content of single red blood cells can be studied without interference of signals from other constituents such as lipids, DNA or the cytoplasm. Resonance Raman studies have been performed to study neuroglobin $(\mathrm{Ngb})$ in its pure form,${ }^{20}$ and in living Escherichia coli bacteria overexpressing $\mathrm{Ngb}^{21}$ However, to our knowledge there is so far no resonance Raman study that has investigated the hemoprotein content of fresh brain tissue. Hundahl et al. recently reported that $\mathrm{Ngb}$ is expressed in a few welldefined nuclei in the rat brain, ${ }^{22}$ one of them being the medial preoptic area. This leads to the assumption that resonance Raman spectroscopy may be used to reveal the hemoprotein content of the medial preoptic nucleus (MPN). Our hypothesis is that investigations of thin brain slices with respect to different hemoprotein contents can enable studies on how neuroglobin (Ngb), hemoglobin $(\mathrm{Hb})$ or other hemoproteins such as cytochrome $\mathrm{c}$ influence cell signalling under stressful situations like oxygen deprivation. 
The aim of this study was to assess the biochemical properties, e.g. composition and hemoprotein structure, of fresh, non-fixed brain tissue with in vitro resonance Raman spectroscopy under close to physiological conditions. To test the sensitivity of the method, two groups of male Sprague-Dawley rats were studied; one group was under normal diet and the other group was under a low iron diet that was supposed to decrease the hemoprotein content in the MPN. The resonance excitation wavelength of $514.5 \mathrm{~nm}$ was used and compared with spectra taken with the NIR excitation wavelength $785 \mathrm{~nm}$ to see if resonance with hemoproteins could be achieved. Furthermore, the goal was to distinguish between different hemoproteins with focus on $\mathrm{Ngb}$.

\section{Materials and Methods}

\section{Rats and dietary treatment}

Ethical approval of the procedures described was given by the regional ethics committee for animal research, "Umeå djurförsöksetiska nämnd", approval No. A121-10.

Eight weaned male Sprague-Dawley rats (age 26-28 days) were randomly divided into two dietary treatment groups: iron-deficient (ID) and control (C). The ID group was fed with the low iron diet $(<5 \mathrm{~g} / \mathrm{kg}$; ABdiets, Netherlands, Reference diet No 4068.30) and the control group was fed with the iron-adequate diet (35 g/kg; SDS, UK) for 11, 12 and 15 days. The diets met all nutritional requirements except for the low iron content of the ID diet. The time span of the ID diet was chosen to last for 11, 12 and 15 days in order to avoid anemia, ${ }^{23}$ since it has been shown that the hemoprotein levels in the brain decrease proportionally to the iron content in tissue before anemia is developed. $^{24}$ Animals were maintained in groups under standard environmental conditions with a 12-h light/dark cycle at a temperature of $21^{\circ} \mathrm{C}$ with humidity and ventilation automatically controlled. All rats were provided free access to food and water. Average food intake was constantly controlled. The growth of ID animals was significantly slower than of $\mathrm{C}$ animals (weight approximately 50\%, data not shown), but anorexia could be ruled out. Poor growth coupled to iron deficiency is commonly observed in animal studies and associated with a poor feed efficiency. ${ }^{25}$

Tissue preparation

Rats were killed by decapitation without anesthetics. Then, the brain was rapidly removed and placed in pre-oxygenated incubation solution at $\leq 4^{\circ} \mathrm{C}\left(150 \mathrm{mM} \mathrm{NaCl}, 5 \mathrm{mM} \mathrm{KCl}, 2 \mathrm{mM} \mathrm{CaCl}_{2}, 10 \mathrm{mM}\right.$ HEPES, $10 \mathrm{mM}$ glucose, $4.93 \mathrm{mM}$ Trizma-base). Coronal $300 \mu \mathrm{m}$ thick slices containing the preoptic area were prepared using a vibroslicer (752M Vibroslice, Campden Instruments, Leicestershire, UK). During slicing, the chamber was filled with the incubation solution $\left(\leq 4^{\circ} \mathrm{C}\right)$. Thereafter, the slices were incubated at 25- 
$27^{\circ} \mathrm{C}$ in oxygenated incubation solution. Before measurements slices were transferred to a glass vessel (Camden Instruments ltd, Leicestershire, UK). The anatomical location of the scanned MPN area was visually identified and assessed with reference to the atlas by Swanson (level 20-22) ${ }^{26}$ by an expert (author E.M.) with many years of experience in electrophysiological recordings of medial preoptic neurons. The MPN is sexually dimorphic and several times larger in males than in females in all vertebrate species. ${ }^{27}$ To get more valuable information on the Raman scattering of hemoproteins from a larger area male rats were used in this study. The rats were studied after 11 (one C, one ID), 12 (two C, two ID) and 15 (one C, one ID) days of diet. One fresh brain tissue slice was used for each Raman mapping scan and for each wavelength, which resulted in two tissue slices per rat.

\section{Raman spectroscopic measurements}

Two different excitation wavelengths were used, the heme sensitive resonance excitation wavelength of $514.5 \mathrm{~nm}$ and the NIR $785 \mathrm{~nm}$ excitation wavelength. A Raman microscope (inVia, Renishaw plc., Gloucestershire, UK) with a $785 \mathrm{~nm}$ excitation laser (max. output power $300 \mathrm{~mW}, \sim 200 \mathrm{~mW}$ at the microscope objective, calculated irradiance $20 \mathrm{MW} / \mathrm{cm}^{2}$, resolution $\sim 2$ $\mathrm{cm}^{-1}$ ), a $514.5 \mathrm{~nm} \mathrm{Ar}^{+}$laser (max. output power $150 \mathrm{~mW}, \sim 75 \mathrm{~mW}$ at the microscope objective, calculated irradiance $\sim 90 \mathrm{~kW} / \mathrm{cm}^{2}$ ), and an upright metallurgic microscope (Leica Microsystems $\mathrm{GmbH}$, Wetzlar, Germany) equipped with a $50 \times$ long working distance objective (CFI L an EPI SLWD $50 \times$, Nikon Instruments Inc, Melville, NY, USA) was used. The spectrometer was calibrated with a Si sample every day and after change of wavelength. No pre-treatment analysis was performed. The static spectral interval of 610-1710 $\mathrm{cm}^{-1}$ was selected for $785 \mathrm{~nm}$ excitation, and $520-1700 \mathrm{~cm}^{-1}$ for $514.5 \mathrm{~nm}$ excitation. $100 \%$ power at $10 \mathrm{~s}$ integration time was applied for all measurements. To get the best result without damaging the sample the focus of the laser beam was set $10 \mu \mathrm{m}$ above the sample by the motorized stage. The laser first penetrated physiological buffer before reaching the buffer immersed tissue. The MPN region of the brain slices were scanned by programming the motorized XYZ-stage (Wire software program, Renishaw plc., Gloucestershire, UK) in a raster scheme according to fig. 1. The scanned areas were approximately $1 \times 1 \mathrm{~mm}$ and the step size $100 \mu \mathrm{m}$, resulting in a total of around 100 spectra per slice.

\section{Raman data processing and analysis}

After examination and approval of the raw Raman data, a number of consecutive preprocessing steps were applied to the raw spectra. Spectra with artifacts (i.e. cosmic rays) were excluded. Smoothing was employed using Eilers' algorithm ${ }^{28}$ with $\mathrm{d}=2$ and $\lambda=10$, and the spectra were then binned to a wavenumber vector with spectral resolution of $1 \mathrm{~cm}^{-1}$. The spectral 
background was subtracted by fitting a piecewise polynomial to each spectrum. ${ }^{29}$ The spectra were vector normalized so that the integrated intensities were equalized. Cluster analysis was performed on the complete preprocessed data set for each wavelength. A hierarchical cluster analysis algorithm using Ward's linkage to merge observations (spectra) similar to each other automatically divided the spectra into a preselected number of groups $;^{30}$ in this study five groups where chosen. The groups were compared by studying their mean spectra.

The $514.5 \mathrm{~nm}$ excitation wavelength was analyzed in more detail since it provided further information about the hemoproteins. This data set consisted of 767 spectra (in total 780 spectra, 13 excluded due to cosmic rays). To compare the ID and $\mathrm{C}$ rats the mean spectrum for each brain slice was calculated after excluding spectra with cosmic rays and spectra from one of the cluster analysis groups that had very pronounced $\mathrm{Hb}$ signals. Difference spectra were calculated from mean spectra as ID - C, from rats with equal duration of diet. To study the content of Ngb the mean intensity of the peak at $1361 \mathrm{~cm}^{-1}$ was calculated for each brain slice, since this Raman line is a prominent Ngb feature. ${ }^{20}$ An ANOVA-test followed by Tukey-Kramer's multiple comparison test were performed to assess whether differences were statistically significant $(p<0.05)$. The ANOVA assumptions of normality and homoscedasticity were checked by using the Lilliefors test ${ }^{31}$ for normality and assessing normality plots and box plots.

\section{Results}

\section{Examination of photo-induced effects}

The raw data from all maps were carefully examined during and after experiment regarding possible photo-induced effects by checking the fluorescent background, and decrease or alteration of the Raman signal and change in color or dryness in the tissue slice. No changes caused by the measurement were observed.

\section{Examination of the resonance effect of $514.5 \mathrm{~nm}$ on the MPN tissue}

Fig. 2 shows typical preprocessed mean Raman spectra of the brain slices for both excitation wavelengths from a $\mathrm{C}$ animal (39 d. of age), the results for the other animals was identical for the $\mathrm{C}$ animals (data not shown). The mean of the tissue mapping scans of the $785 \mathrm{~nm}$ excitation wavelength showed a broad change of the Raman intensity between $1300-1670 \mathrm{~cm}^{-1}$ but the hemoglobin signals were rather weak, and from this data we could not distinguish between different hemoproteins or their states. Therefore, this wavelength was not analyzed further. The mapping scans from the $514.5 \mathrm{~nm}$ Raman line were in clear resonance with hemoproteins and showed heterogeneous features regarding the hemoprotein composition and intensity, as discussed below. 
Table 1 shows previously published Raman line assignments ${ }^{20,32,33}$ of the mean spectra seen in fig. 2. The main differences between the mean spectra derived with the two different wavelengths lye in the Raman signal intensity of the hemoprotein in the intervals $1200-1400 \mathrm{~cm}^{-1}$ and $1550-1640$ $\mathrm{cm}^{-1}$.

Table I. Raman line assignment of the MPN tissue slices. ${ }^{15,20,32,33}$

\begin{tabular}{|c|c|c|}
\hline $\begin{array}{l}785 \mathrm{~nm} \text { Raman } \\
\text { lines }\left[\mathrm{cm}^{-1}\right]\end{array}$ & Assignment & $\begin{array}{l}\text { Prominent } 514.5 \mathrm{~nm} \\
\text { Raman lines }\left[\mathrm{cm}^{-1}\right] \\
\end{array}$ \\
\hline 1661 & amide I & \\
\hline- & heme $v_{10}$ oxy & 1640 (weak shoulder) \\
\hline $\begin{array}{l}1624 \quad \text { (weak } \\
\text { shoulder) }\end{array}$ & heme $v(C=C)$ vinyl & 1624 \\
\hline $\begin{array}{l}1607 \quad \text { (weak } \\
\text { shoulder) }\end{array}$ & heme $v_{10}$ deoxy & 1607 \\
\hline 1585 (weak) & heme $v_{37}$ & 1585 \\
\hline 1554 & heme $v_{11}$ & 1554 \\
\hline 1505 (weak) & $\begin{array}{l}\text { heme } v_{3} \text { (six-coordinate, low } \\
\text { spin) }\end{array}$ & 1470,1493 or 1505 \\
\hline 1448 & $\delta\left(\mathrm{CH}_{2} / \mathrm{CH}_{3}\right)$ & 1448 \\
\hline 1439 & $\begin{array}{l}\mathrm{CH}_{2} \text { deformation mode from } \\
\text { proteins and lipids }\end{array}$ & \\
\hline 1399 (weak) & heme $v_{20}$ & 1399 \\
\hline- & heme $v_{4}$ & $\begin{array}{l}1374 \text { (oxy) and } 1356 \\
\text { or } 1361 \text { (deoxy) strong }\end{array}$ \\
\hline $\begin{array}{l}1339 \quad \text { (weak } \\
\text { shoulder) }\end{array}$ & heme $v_{41}$ & 1339 \\
\hline 1333 & $\begin{array}{l}\mathrm{CH}_{3} / \mathrm{CH}_{2} \text { twisting of proteins } \\
\text { and nucleic acids }\end{array}$ & \\
\hline- & heme $v_{21}$ & 1309 \\
\hline 1299 & $\begin{array}{l}\text { twisting and wagging } \\
\text { vibrations from fatty acids and } \\
\text { cholesterol }\end{array}$ & \\
\hline 1269 & amide III and lipids & \\
\hline- & heme $v_{13}$ & 1231 \\
\hline
\end{tabular}




\begin{tabular}{|l|l|l|}
\hline 1206 & tyrosine & \\
\hline 1172 & cytosine or heme $v_{30}$ & 1172 \\
\hline 1156 & C-C and C-N proteins & \\
\hline 1126 & $v(\mathrm{c}-\mathrm{c})$ from lipids & \\
\hline 1086 & $\begin{array}{l}v(\mathrm{c}-\mathrm{c}) \text { stretching and } \mathrm{PO}_{2} \\
\text { symmetric stretching }\end{array}$ & \\
\hline 1061 & $v(\mathrm{c}-\mathrm{c})$ from lipids & \\
\hline 1030 & phenylalanine & \\
\hline 1004 & phenylalanine & \\
\hline
\end{tabular}

Raw spectra acquired with the $514.5 \mathrm{~nm}$ excitation wavelength were diverse but showed three main different types: spectra with a prominent hemoglobin content (fig. 3a), spectra revealing Raman lines assigned to tissue and $\mathrm{Ngb}$ content (fig. 3b), and spectra with mainly tissue and less Ngb signals (fig. 3c). Clearly, resonance with the hemoprotein content of the MPN was achieved with the $514.5 \mathrm{~nm}$ laser. The observations made from the raw data were confirmed by the cluster analysis. Spectra with high $\mathrm{Hb}$ signals were collected in group 5, and spectra with higher tissue and Ngb content were categorized into group 1 - 4, with increasing Ngb signal content going from 1 to 4 , as can be seen in fig. 4 . The figure also contains an inset of the five cluster analysis groups from the $785 \mathrm{~nm}$ excitation wavelength in the region with the most prominent differences $\left(1300-1630 \mathrm{~cm}^{-1}\right)$. Note that the differences between the groups of $785 \mathrm{~nm}$ lie in the intensity profile but not in shift or changes in the Raman lines. The distribution of the different groups can be seen in fig. 5. Spectra with high $\mathrm{Hb}$ contents (group 5) came from single scanning points randomly distributed in all tissues. Groups $1-4$ were more evenly distributed over the MPN tissue slices (fig. 5).

The hemoprotein bands could be analyzed in detail by studying the preprocessed mean spectra of the cluster analysis groups, as shown in fig. 4 .

The uppermost spectrum of fig. 4 (group 5) shows a typical $\mathrm{Hb}$ spectrum, where both the ferrous five-coordinated high spin state (deoxy) and the ferric six-coordinated low spin state (oxy) are present. The mixed oxygenation state of $\mathrm{Hb}$ is expected when looking at fresh, non-fixed, and untreated tissue. The spectra from group $1-4(\mathrm{n}=22, \mathrm{n}=285, \mathrm{n}=149, \mathrm{n}=247)$ show Raman spectra of different mixtures of the ferrous six-coordinated low spin state and the six-coordinated ferric low spin form that are typical for $\mathrm{Ngb} .^{20}$

Examination of effects from the iron deficiency diet (514.5 nm) 
The difference spectra of the mean data of the MPN tissue scans were compared, see fig. 6. The difference spectra were taken from ID minus C animals of same diet duration. The differences were further examined by studying the intensities (I) of the $v_{4}$ Raman line at $1361 \mathrm{~cm}^{-1}$ for the $\mathrm{C}$ and the ID rats, see fig. 7. The ANOVA and Tukey-Kramer tests showed that except for ID diet for 12 days (I) the ID rats showed a statistically significant decrease $(p<0.05)$ of hemoprotein. The Lilliefors test and assessment of normality/box plots (not shown) showed no large deviations from normality and homoscedasticity. When calculating the mean intensity for each group of rats and taking the ratio $\left(\mathrm{I}_{\mathrm{ID}} / \mathrm{I}_{\mathrm{C}}\right)$ an overall decrease of $\mathrm{Ngb}$ content of approximately $20 \%$ was observed due to the ID diet.

\section{Discussion}

In vitro resonance micro-Raman spectra from the MPN region of the anterior hypothalamus from male Sprague Dawley rats have here been presented for the first time. High quality resonance Raman spectra were achieved in the mapping mode on thin, fresh brain slices of the MPN. In contrast to the $785 \mathrm{~nm}$, the $514.5 \mathrm{~nm}$ excitation wavelength gave detailed information on hemoprotein species and their states. When applying resonance Raman spectroscopy it is important to avoid possible photo-induced effects caused by the excitation laser. ${ }^{34,35}$ In this study, the thin fresh tissue slice was put in a glass-vessel and covered with physiological buffer. The tissue was always covered in buffer solution, and no fixation was applied. The focus was set $10 \mu \mathrm{m}$ above the tissue in order to avoid direct focus on the tissue. The irradiance applied was calculated to $90 \mathrm{~kW} / \mathrm{cm}^{2}$, which is comparable to a previous study where photo-induced effects on single erythrocytes were studied. $^{36}$ In that study it was shown that this irradiance $\left(90 \mathrm{~kW} / \mathrm{cm}^{2}\right)$ can cause a conversion of $\mathrm{oxyHb}$ to the metHb after $160 \mathrm{~s}$. Note, the integration time applied here was 10 seconds; thereafter the defocused laser spot was moved $100 \mu \mathrm{m}$ to the next point in the mapping raster. Only one spectrum was taken per point and the tissue slices were only used for one mapping scan. When applying the NIR-illumination, the irradiance was calculated to 20 $\mathrm{MW} / \mathrm{cm}^{2}$, which can be considered high compared to a previous study. ${ }^{37}$ However, in the study presented here, the irradiance is over-estimated since the calculation did not include defocusing of the laser beam and due to the scattering of the laser light in the buffer covering the tissue; accordingly, no photo-induced effects due to laser illumination or drying out of the tissue could be detected in the spectral raw data.

The vibrational modes of the chromophoric heme group were selectively enhanced by the $514.5 \mathrm{~nm}$ excitation wavelength. It is well known that this provides valuable information of conformational changes and/or changes in the spin and oxidation state of the iron. For instance, when the heme is in a five-coordinated ferrous high spin state, the $v_{4}$ band is found at 
$1356 \mathrm{~cm}^{-1}$ and the $v_{3}$ band at $1470 \mathrm{~cm}^{-1}$, which is typical for $\mathrm{Hb}^{35}$ In the ferrous six-coordinated low spin form the $v_{4}$ band is found at $1361 \mathrm{~cm}^{-1}$ and $v_{3}$ at $1493 \mathrm{~cm}^{-1}$, which is characteristic for $\mathrm{Ngb}^{20}$ For the six-coordinated ferric low spin form, the $v_{4}$ Raman line shifts to $1374 \mathrm{~cm}^{-1}$, and the $v_{3}$ Raman line to $1505 \mathrm{~cm}^{-1} \cdot 20,38$

Most interestingly, the $v_{4}$ band was predominantly here found at 1361 $\mathrm{cm}^{-1}$ as seen in the raw spectra shown in fig. $3 \mathrm{~b}$ and c, which indicates the sixcoordinated deoxy state typical for $\mathrm{Ngb}$. This was verified by the $v_{3}$ line at $1493-1505 \mathrm{~cm}^{-1}$ which also showed the hexa-coordinated low spin state. ${ }^{20,21}$ Hence, we concluded that these resonance Raman spectra originated from $\mathrm{Ngb}$. The hexa-coordinated feature may have originated from hemochrome, a derivative of $\mathrm{Hb}$, often caused by dehydration of the sample or the usage of fixatives. ${ }^{39}$ However, since the tissue was immersed in buffer and no fixation protocol was used, this can be ruled out. For the prominent Hb spectra, the $v_{10}$ band was shown both at 1607 and $1640 \mathrm{~cm}^{-1}$, which indicated both the oxy-, and the deoxygenation state.

The appropriate number of groups for cluster analysis cannot be determined beforehand, because it depends on the dataset at hand and the differences that are to be studied. ${ }^{30}$ Bird et al. used hierarchical cluster analysis and found that five groups were suitable for reproducing the histological architecture of lymph nodes, ${ }^{40}$ indicating that this could be a reasonable choice. Here, cluster analysis was performed with three to nine groups, and five groups were considered appropriate for studying different hemoprotein species and conformational states in fresh brain tissue. The five groups of the cluster analysis data for the resonance Raman spectra $(\lambda=514.5 \mathrm{~nm})$ are shown in Figs. 4 and 5. Group 5 consisted of typical Hb Raman lines and group 1-4 consisted of typical Ngb and tissue Raman lines with increasing Ngb content (fig. 4). The distribution of the groups (fig. 5) showed that group 5 only appeared at certain spots most likely coming from small blood vessels, whereas groups 1-4 were more evenly distributed over the tissue. These results show that different hemoprotein types, e.g. $\mathrm{Hb}$ and $\mathrm{Ngb}$, can be distinguished in fresh tissue in vitro using resonance Raman spectroscopy. As can be seen in the inset of fig. 4 the cluster analysis on the data from $785 \mathrm{~nm}$ excitation wavelength showed changes in the intensity profile between 1300 to $1630 \mathrm{~cm}^{-1}$. In many studies intensity differences are used to differentiate between different tissue types, ${ }^{15}$ here the aim was to reveal clear hemoglobin species and states. Only the strongest hemoprotein lines for the NIR excitation were revealed $^{33}$ and no comparison between different hemoprotein contents or their oxidation and spin states could be gained. This shows that resonance Raman spectroscopy with the $514.5 \mathrm{~nm}$ excitation line is necessary if different hemoproteins are to be studied in soft brain tissue. 
Resonance Raman spectroscopy was here used to study the effect of a low iron diet compared to normal diet. The difference spectra (ID-C) in fig. 6 showed that ID rats presented a decreased amount of amide I $\left(1661 \mathrm{~cm}^{-1}\right)$ and lipids $\left(1441,1457,1445,1422 \mathrm{~cm}^{-1}\right)$. Furthermore, the difference in the hemoprotein intensity is decreased $\left(1550-1650 \mathrm{~cm}^{-1}\right.$ and $\left.1200-1470 \mathrm{~cm}^{-1}\right)$. The ID diet decreased the Ngb content, as can be seen in fig. 7, where the intensity distribution of the $v_{4}$ band at $1361 \mathrm{~cm}^{-1}$ is shown. The difference between ID and $\mathrm{C}$ was statistically significant $(p<0.05)$, and indicated a general decrease of hemoprotein content of approximately $20 \%$ for a diet that lasted 11-15 days.

In conclusion, the results show that resonance Raman spectroscopy with the $514.5 \mathrm{~nm}$ excitation line is very well suited for hemoprotein studies of the MPN. Especially the possibility to detect Ngb by resonance Raman spectroscopy is most exciting.

\section{Conclusion}

This study demonstrates that high quality in vitro resonance Raman spectroscopic data can be routinely acquired from fresh brain tissue samples. The resonance Raman data indicated the presence of Ngb throughout the whole MPN of all tissue slices, also in the group with lowest Ngb content. This was not observed by the NIR Raman spectra; only the strongest hemoprotein Raman lines at this wavelength were revealed but the $v_{4}$ line was absent. Hemoglobin could be mainly attributed to a few distinguished places most likely coming from small blood vessels. Iron-deficient MPN samples were characterized by a decrease of the hemoprotein content compared to the iron-adequate MPN samples. We conclude that a short-term low-iron diet caused changes in the Ngb content of hypothalamic tissue. The results are promising for the future investigations of hemoproteins and their role in neuro-protection.

\section{Acknowledgements}

This work was supported by the Swedish Research Council, the European Union Structural fund, objective 2, Norra Norrland; the Swedish Brain Foundation; and the Kempe Foundation. The authors acknowledge the Vibrational Spectroscopy Platform (ViSP) at Umeå University and thank Dr. Thomas Wågberg and Dr. András Gorzsás, Umeå University, Sweden for valuable input and help with the Raman measurements, Prof Staffan Johansson and Dr Michael Druzin, Department of Integrative Medical Biology, Umeå University, Sweden, for valuable discussions.

\section{References}

1. Chan JW, Taylor DS, Lane SM, Zwerdling T, Tuscano J, Huser T. Nondestructive identification of individual leukemia cells by laser trapping Raman spectroscopy. Analytical Chemistry 2008;80(6): 2180-87. 
2. Chan JW, Taylor DS, Zwerdling T, Lane SM, Ihara K, Huser T. Micro-Raman spectroscopy detects individual neoplastic and normal hematopoietic cells. Biophysical Journal 2006;90(2): 648-56.

3. Crow P, Barrass B, Kendall C, et al. The use of Raman spectroscopy to differentiate between different prostatic adenocarcinoma cell lines. British Journal of Cancer 2005;92(12): 2166-70.

4. Crow P, Molckovsky A, Stone N, Uff J, Wilson B, Wongkeesong LM. Assessment of fiberoptic near-infrared Raman spectroscopy for diagnosis of bladder and prostate cancer. Urology 2005;65(6): 1126-30.

5. Ferlay J, Autier P, Boniol M, Heanue M, Colombet M, Boyle P. Estimates of the cancer incidence and mortality in Europe in 2006. Annals of Oncology 2007;18(3): 581-92.

6. Guo JY, Du B, Qian M, Cai WY, Wang ZG, Sun ZR. Raman spectroscopic identification of normal and malignant hepatocytes. Chinese Optics Letters 2009;7(1): 60-63.

7. Haka AS, Shafer-Peltier KE, Fitzmaurice M, Crowe J, Dasari RR, Feld MS. Diagnosing breast cancer by using Raman spectroscopy. Proceedings of the National Academy of Sciences of the United States of America 2005;102(35): 12371-76.

8. Harvey TJ, Faria EC, Henderson A, et al. Spectral discrimination of live prostate and bladder cancer cell lines using Raman optical tweezers. Journal of Biomedical Optics 2008;13(6).

9. Mahadevan-Jansen A, Richards-Kortum RR. Raman spectroscopy for the detection of cancers and precancers. Journal of Biomedical Optics 1996;31.

10. Koljenovic S, Schut TCB, Wolthuis R, et al. Raman spectroscopic characterization of porcine brain tissue using a single fiber-optic probe. Analytical Chemistry 2007;79(2): 55764.

11. Tay LL, Tremblay RG, Hulse J, Zurakowski B, Thompson M, Bani-Yaghoub M. Detection of acute brain injury by Raman spectral signature. Analyst;136(8): 1620-26.

12. Beleites C, Geiger K, Kirsch M, Sobottka SB, Schackert G, Salzer R. Raman spectroscopic grading of astrocytoma tissues: using soft reference information. Analytical and Bioanalytical Chemistry 2011;400(9): 2801-16.

13. Meyer T, Bergner N, Bielecki C, et al. Nonlinear microscopy, infrared, and Raman microspectroscopy for brain tumor analysis. Journal of Biomedical Optics 2011;16(2).

14. Kirsch M, Schackert G, Salzer R, Krafft C. Raman spectroscopic imaging for in vivo detection of cerebral brain metastases. Analytical and Bioanalytical Chemistry 2010;398(4): 1707-13.

15. Beljebbar A, Dukic S, Amharref N, Manfait M. Ex vivo and in vivo diagnosis of C6 glioblastoma development by Raman spectroscopy coupled to a microprobe. Analytical and Bioanalytical Chemistry 2010;398(1): 477-87.

16. Darvin ME, Sterry W, Lademann J, Vergou T. The Role of Carotenoids in Human Skin. Molecules 2011;16(12): 10491-506.

17. Ogawa M, Harada Y, Yamaoka Y, Fujita K, Yaku H, Takamatsu T. Label-free biochemical imaging of heart tissue with high-speed spontaneous Raman microscopy. Biochemical and Biophysical Research Communications 2009;382(2): 370-74.

18. Torres IP, Terner J, Pittman RN, Proffitt E, Ward KR. Measurement of hemoglobin oxygen saturation using Raman microspectroscopy and 532-nm excitation. Journal of Applied Physiology 2008;104(6): 1809-17. 
19. Spiro TG, Strekas TC. Resonance Raman Spectra of Heme Proteins. Effects of Oxidation and Spin State. Journal of the American Chemical Society 1974;96(2): 338-45.

20. Couture M, Burmester T, Hankeln T, Rousseau DL. The heme environment of mouse neuroglobin - Evidence for the presence of two conformations of the heme pocket. Journal of Biological Chemistry 2001;276(39): 36377-82.

21. Ramser K, Wenseleers W, Dewilde S, Van Doorslaer S, Moens L, Hanstorp D. Microresonance Raman study of optically trapped Escherichia coli cells overexpressing human neuroglobin. Journal of Biomedical Optics 2007;12(4).

22. Hundahl CA, Allen GC, Nyengaard JR, et al. Neuroglobin in the Rat Brain: Localization. Neuroendocrinology 2008;88(3): 173-82.

23. Soliman GZA, Mahfouz MH, Emara IA. Effect of Different Types of Oral Iron Therapy Used for the Treatment of Iron Deficiency Anemia and Their Effects on Some Hormones and Minerals in Anemic Rats. Journal of American Science 2010;6(6): 109-18.

24. Guiang Iii SF, Georgieff MK, Lambert DJ, Schmidt RL, Widness JA. Intravenous iron supplementation effect on tissue iron and hemoproteins in chronically phlebotomized lambs. American Journal of Physiology - Regulatory Integrative and Comparative Physiology 1997;273(6 42-6): R2124-R31.

25. Beard JL, Zhan CCS, Brigham DE. GROWTH IN IRON-DEFICIENT RATS. Proceedings of the Society for Experimental Biology and Medicine 1995;209(1): 65-72.

26. Swanson LW. Brain Maps: Structure of the Rat Brain, Second Edition In: edition ESn, editor, 1999.

27. Gorski RA, Harlan RE, Jacobson CD. Evidence for the existence of a sexually dimorphic nucleus in the preoptic area of the rat. Journal of Comparative Neurology 1980;193(2): 52939.

28. Eilers PHC. A perfect smoother. Analytical Chemistry 2003;75(14): 3631-36.

29. Cao A, Pandya AK, Serhatkulu GK, et al. A robust method for automated background subtraction of tissue fluorescence. Journal of Raman Spectroscopy 2007;38(9): 1199-205.

30. Johnsson RA, Wichern DW. Applied Multivariate Statistical Analysis. Upper Saddle River, NJ:: Prentice-Hall, 1998.

31. Lilliefors HW. On the Kolmogorov-Smirnov test for normality with mean and variance unknown. Journal of the American Statistical Association 1967;62(318): 399-402.

32. Movasaghi Z, Rehman S, Rehman IU. Raman spectroscopy of biological tissues. Applied Spectroscopy Reviews 2007;42(5): 493-541.

33. Wood BR, Caspers P, Puppels GJ, Pandiancherri S, McNaughton D. Resonance Raman spectroscopy of red blood cells using near-infrared laser excitation. Analytical and Bioanalytical Chemistry 2007;387(5): 1691-703.

34. Puppels GJ, Olminkhof JHF, Segers-Nolten GMJ, Otto C, DeMul FFM, Greve J. Laser Irradiation and Raman Spectroscopy of Single Living Cells and Chromosomes: Sample Degradation Occurs with $514.5 \mathrm{~nm}$ but with $660 \mathrm{~nm}$ Laser Light. Experimental Cell Research 1991;195: 361-67.

35. Wood BR, McNaughton D. Raman excitation wavelenghts investigations of single red blood cells in vivo. Journal of Raman Spectroscopy 2002;33: 517-23.

36. Ramser K, Bjerneld EJ, Fant C, Käll M. Importance of substrate and photo-induced effects in Raman spectroscopy of single functional erythrocytes. Journal of Biomedical Optics 2003;8(2): 173-78. 
37. Candefjord S, Ramser K, Lindahl OA. Effects of snap-freezing and near-infrared laser illumination on porcine prostate tissue as measured by Raman spectroscopy. Analyst 2009;134(9): 1815-21.

38. Ramser K, Wenseleers W, Dewilde S, Van Doorslaer S, Moens L, Hanstorp D. A combined micro-resonance Raman and absorption set-up enabling in vivo studies under varying physiological conditions: the nerve globin in the nerve cord of Aphrodite aculeata. Journal of Biochemical and Biophysical Methods 2007;70: 627-33.

39. Asghari-Khiavi M, Mechler A, Bambery KR, McNaughton D, Wood BR. A resonance Raman spectroscopic investigation into the effects of fixation and dehydration on heme environment of hemoglobin. Journal of Raman Spectroscopy 2009;40(11): 1668-74.

40. B. Bird, M. Miljkovic, M.J. Romeo, J. Smith, N. Stone, M.W. George, M. Diem. Infrared Micro-Spectral Imaging: Distinction of Tissue Types in Axillary Lymph Node Histology. BMC Clinical Pathology 2008;8(1): 8.

Figures:

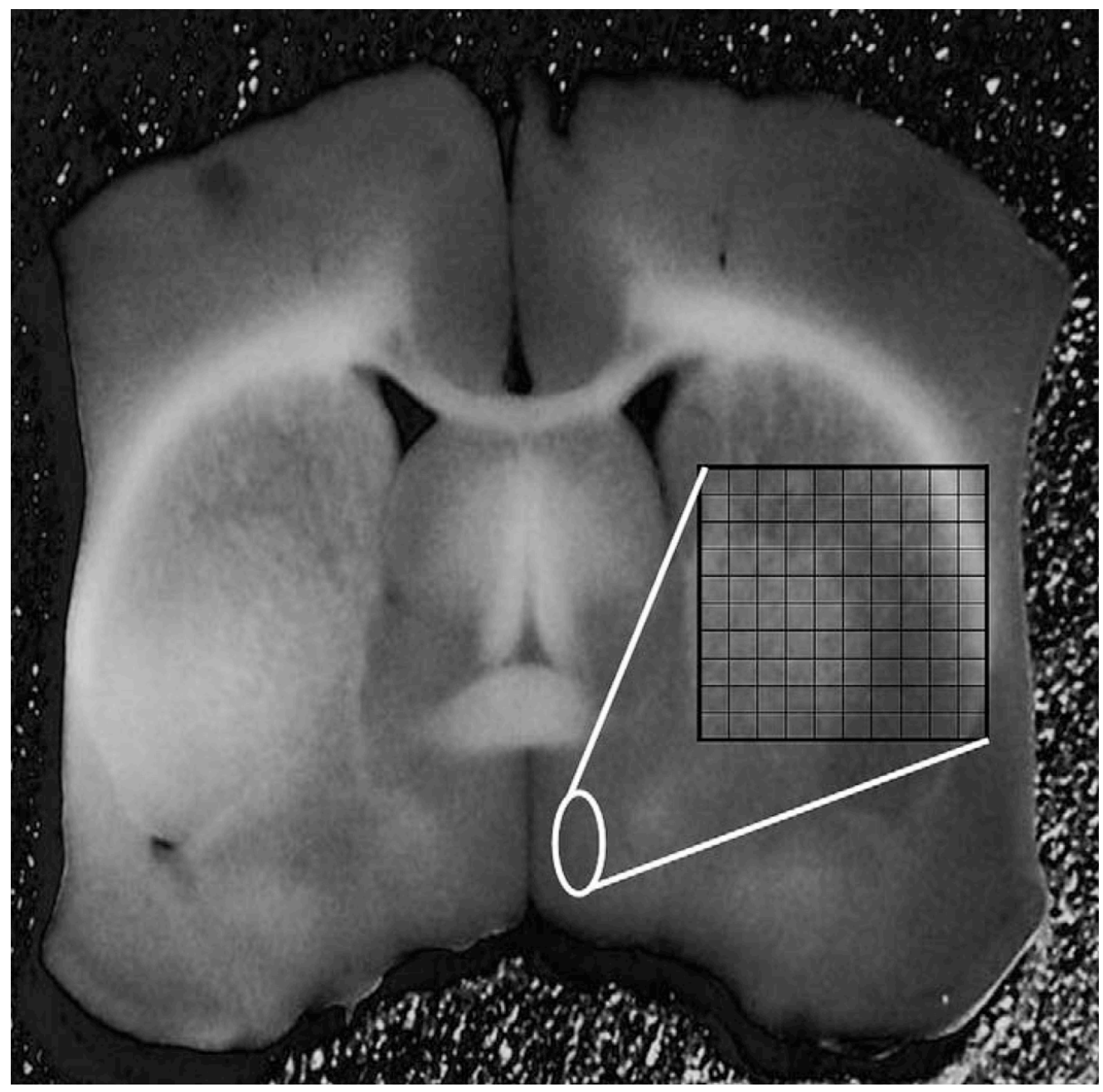


Fig. 1 Schematic photograph of a brain slice from the anterior hypothalamus. The MPN is indicated by the white ellipse. The spectral mapping region is zoomed out as a raster $(\sim 1 \times 1 \mathrm{~mm})$. One spectrum was acquired at each cross-section.

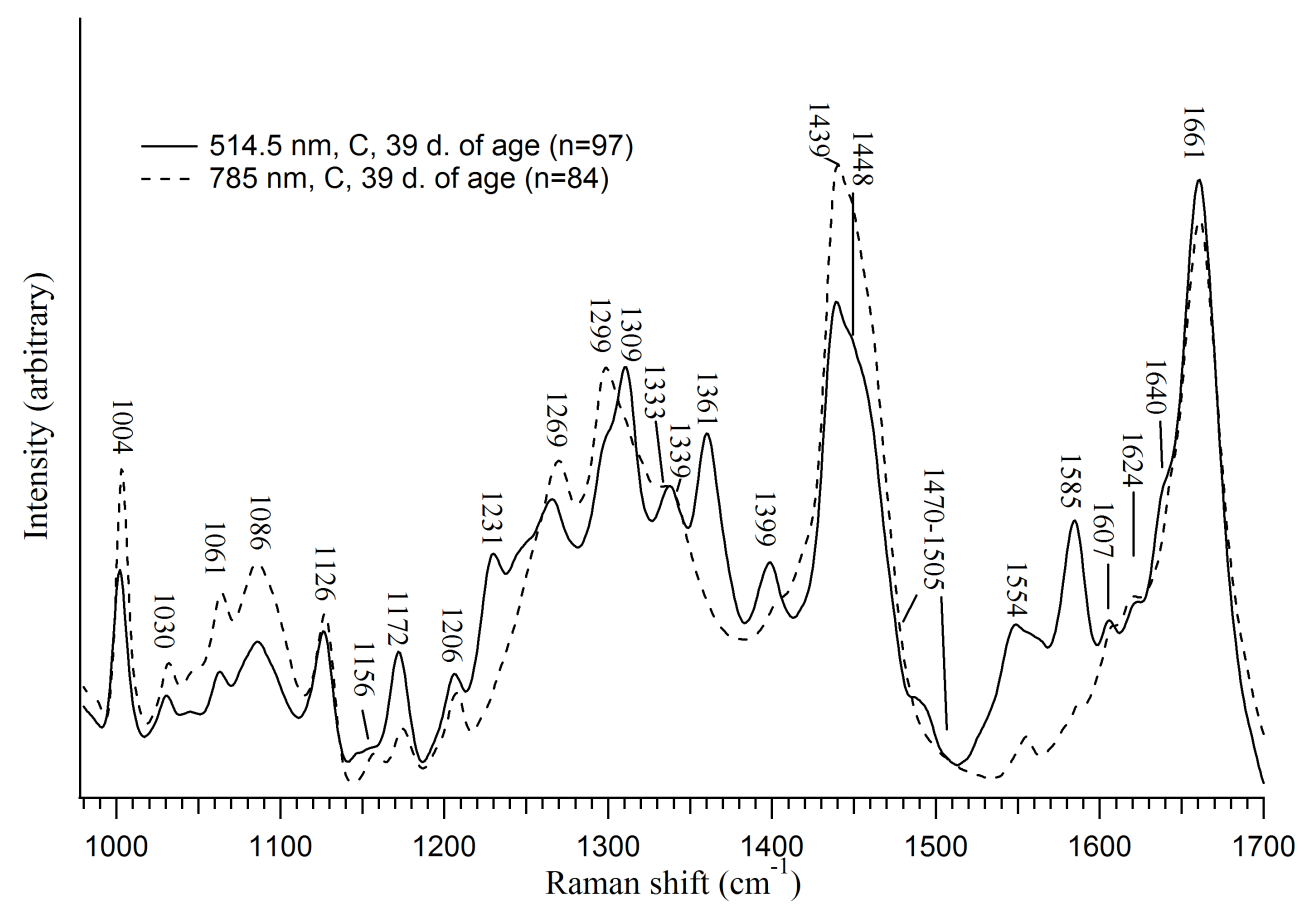

Fig. 2. Mean preprocessed spectra from control animals: dotted line for $785 \mathrm{~nm}$ (number of spectra $n=84$, exclusion of artifacts) and mean spectrum excited with $514.5 \mathrm{~nm}(n=97$ exclusion of artifacts).

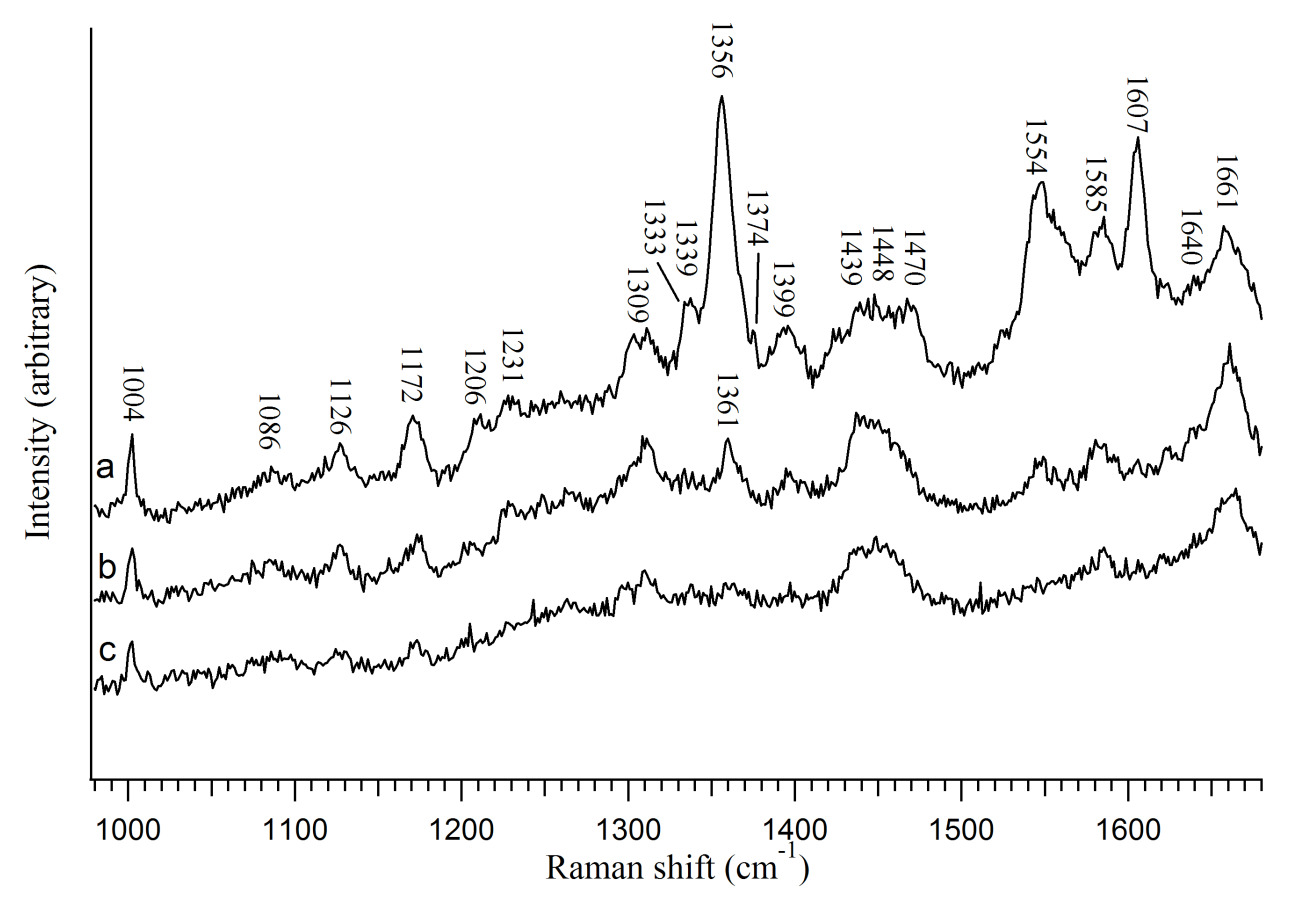


Fig. 3 Raw resonance Raman spectra from control animals, $514.5 \mathrm{~nm}$ laser excitation. a) spectrum from a Hb "spot", b) spectrum with Raman lines from tissue and $\mathrm{Ngb}, c)$ spectrum with prominently tissue and less Ngb signals.

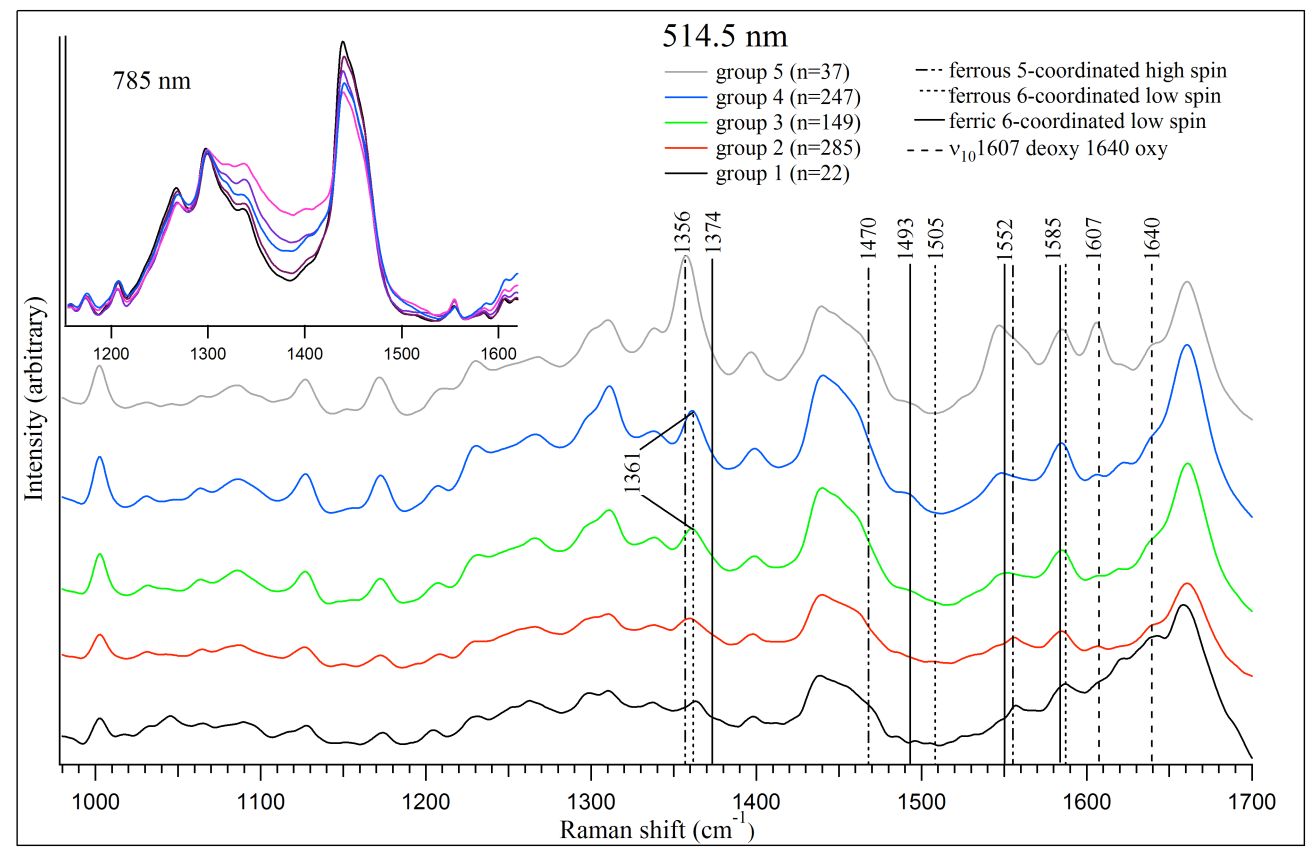

Fig. 4. The results for the cluster analysis of preprocessed spectra acquired at $514.5 \mathrm{~nm}$. The groups have been labeled with different colors: black (group 1), red (group 2) green (group 3), blue (group 4) and gray (group 5). The inset (left upper corner) shows the cluster analysis for $785 \mathrm{~nm}$ for the region of the most prominent differences $\left(1300-1630 \mathrm{~cm}^{-1}\right)$. Please note, the five cluster analysis groups for $785 \mathrm{~nm}$ cannot be compared to $514.5 \mathrm{~nm}$, hence the groups are shown in different colors.
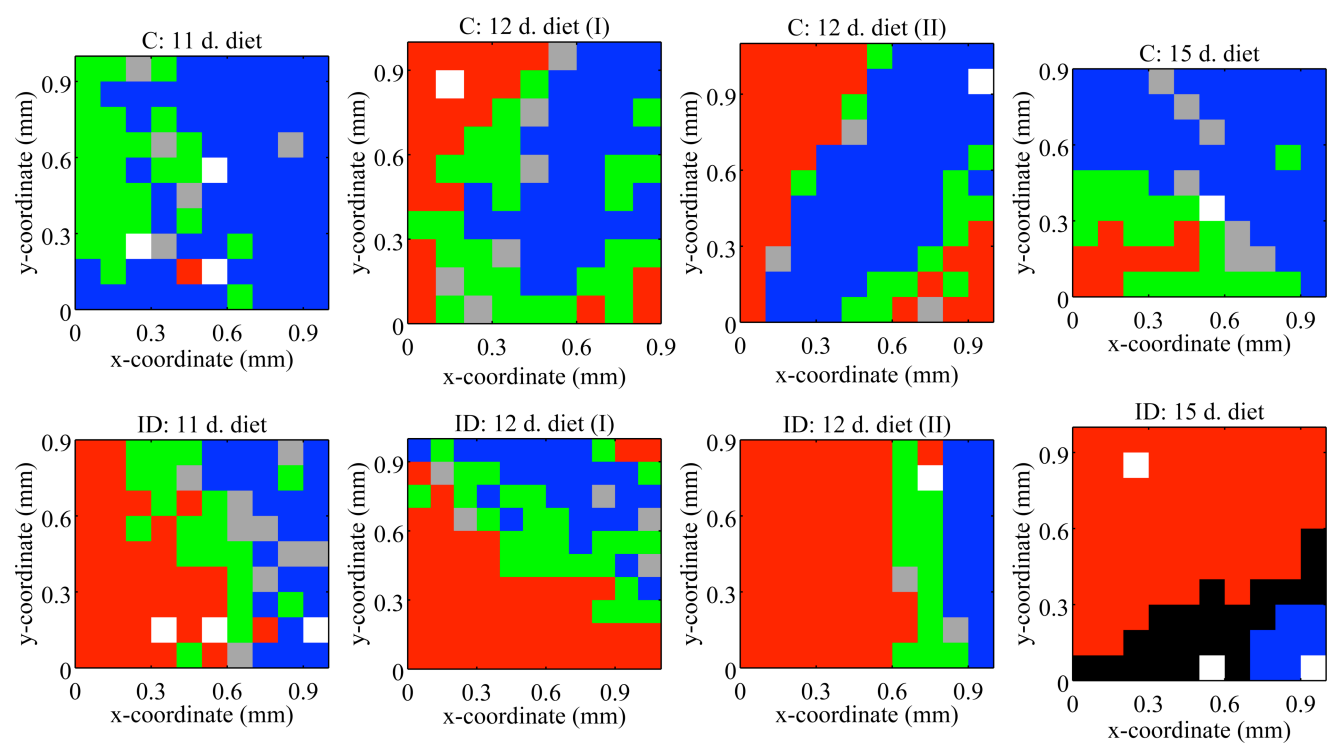

Fig. 5. Cluster analysis for the different tissue slices from $C$ and ID rats. Note the duration of the diet. The white squares denote spectra that were excluded due to artifacts. 


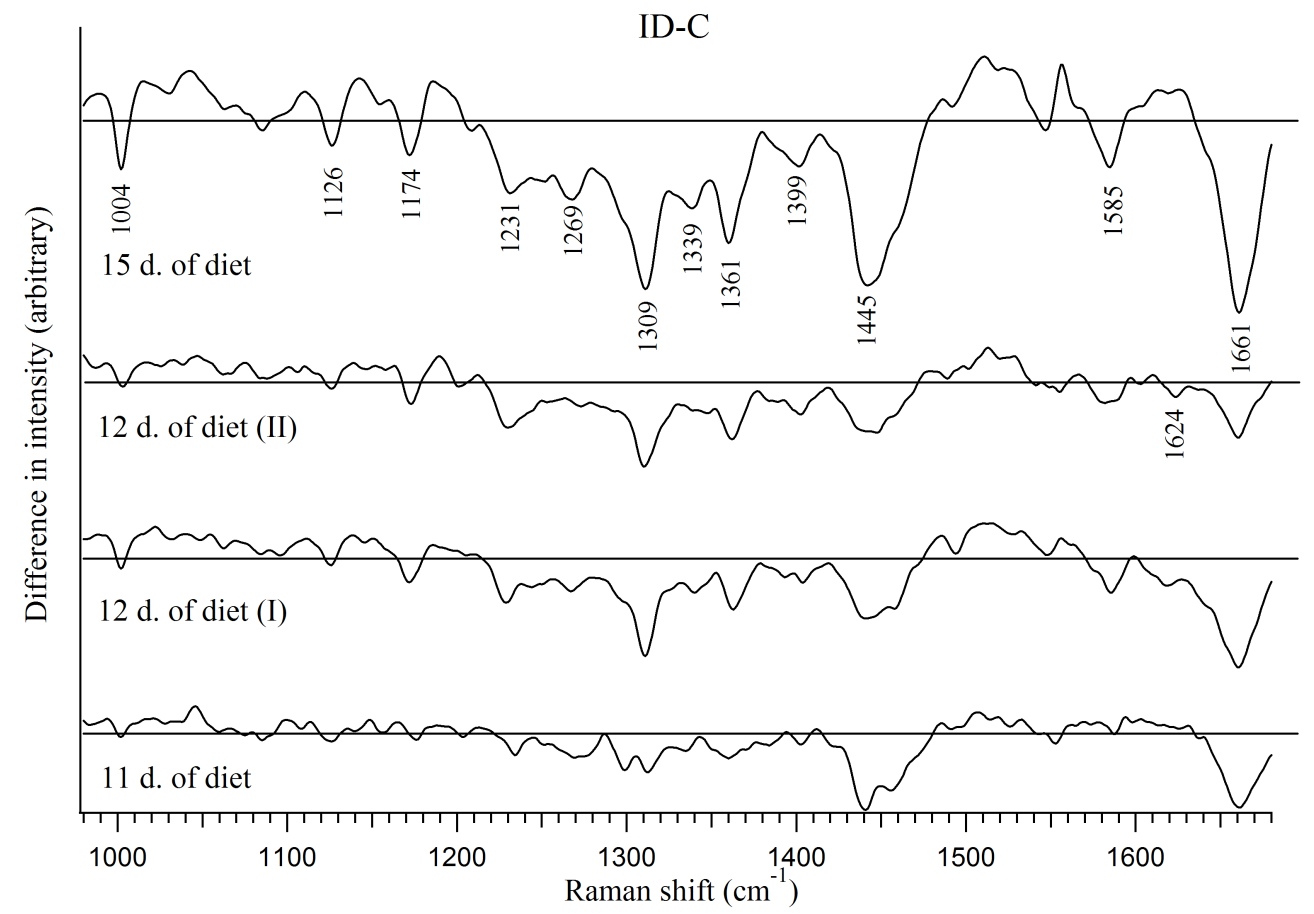

Fig. 6. Difference spectra between ID and C derived from processed mean Raman data for the $514.5 \mathrm{~nm}$ excitation of the brain tissue scans. 


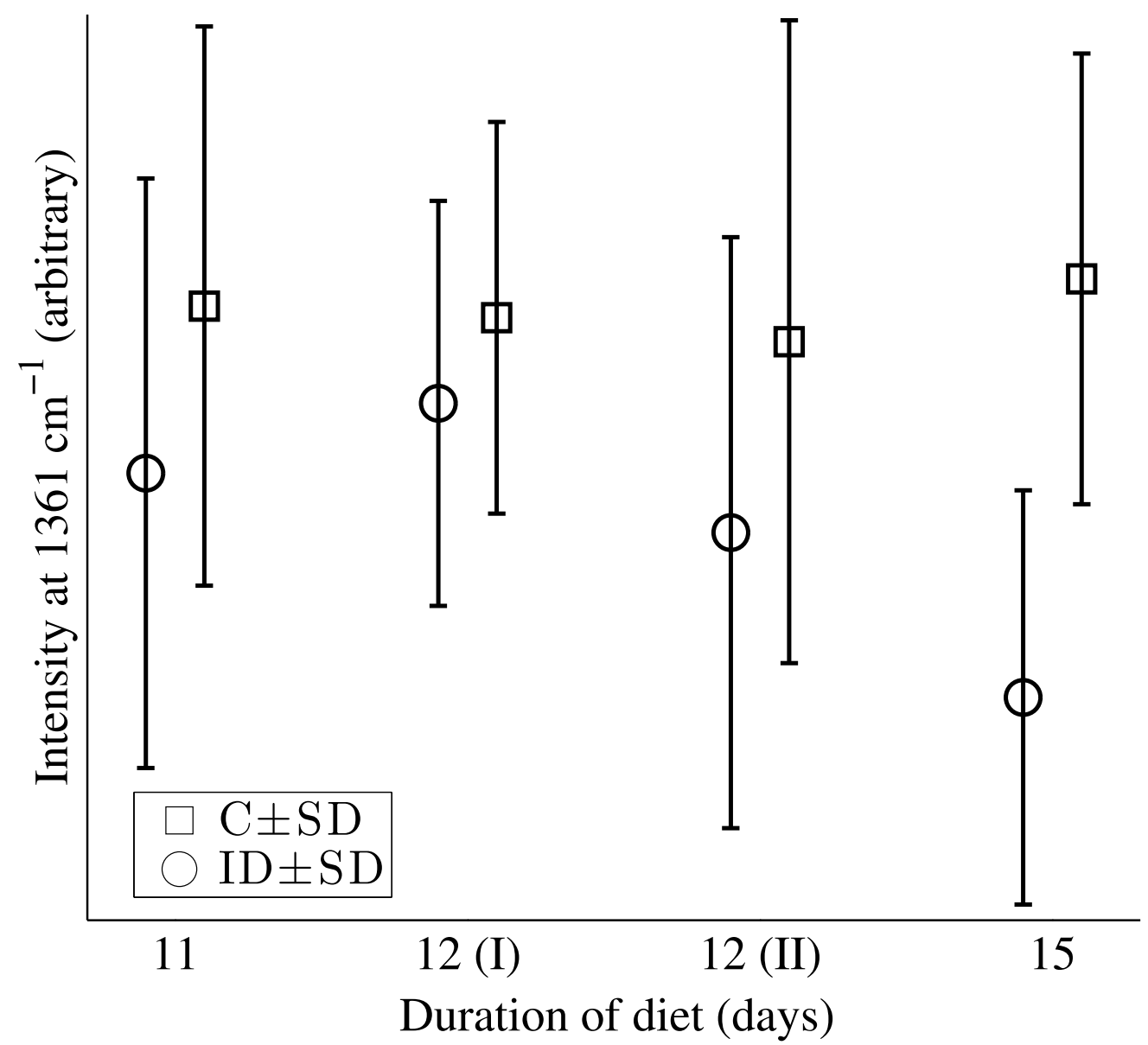

Fig 7. Mean and standard deviation of the intensity at the 04 Raman line at $1361 \mathrm{~cm}^{-1}$ for C and ID rats. 